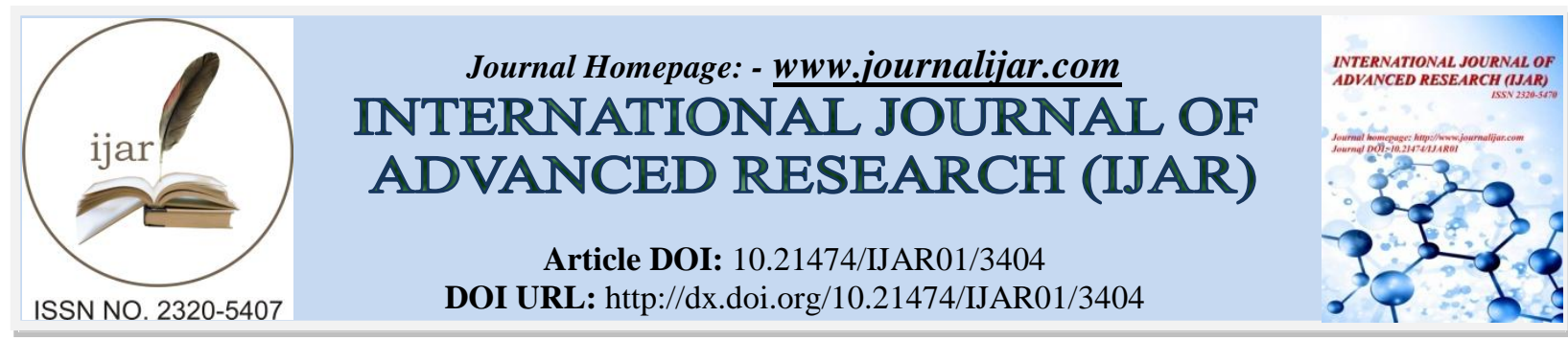

RESEARCH ARTICLE

\title{
INFLUENCES OF NIGELLA SATIVA ON ANTIOXIDANT ACTIVITY IN BROILERS TOXIFIED WITH SYNTHETIZED ORGANOPHOSPHORUS COMPOUNDS.
}

Al Shimaa M Ibrahim ${ }^{1}$, El-Said E Esmail ${ }^{1}$ and Essam S Soliman ${ }^{2 *}$.

1. Department of Chemistry, Faculty of Science, Suez Canal University, Ismailia, Egypt, 41522.

2. Department of Animal Hygiene, Zoonosis \& Animal Behavior, Faculty of Veterinary Medicine, Suez Canal University, Ismailia, Egypt, 41522.

\section{Manuscript Info}

\section{Manuscript History}

Received: 09 December 2016

Final Accepted: 15 January 2017

Published: February 2017

Key words:-

Antioxidant, Broilers, Diazinon, Nigella sativa.

\begin{abstract}
Nigella has historical and religious uses for preventing and treating many different kinds of diseases. Influences of Nigella on bird performance, biochemical, antioxidant and immunological status in birds toxified with synthetized diazinon were studied. 150 broiler chick were received and divided into three groups; G1 and G2 were supplemented with Nigella (1.4 gm / 100 gm; 2.8 gm / 100 gm ration; respectively) and G3 is control. G1 and G2were toxified with synthetized diazinon (100 ppm / $1 \mathrm{~L}$ drinking water). A total of 180 samples (60 serum, 60 plasma and 60 intestinal swab) were collected during the study period. The results revealed the ability of Nigella to maintain biochemical parameters as TP; Alb; Glob; ALT; AST; Urea and Creatinine after prolonged deviation. Glutathione peroxidase was sharply decreased, Glutathione Reductase was sharply increased indicating the stimulation action. Superoxide Dimutase was significantly increased until the $4^{\text {th }}$ week of age followed by significant decrease. Immunoglobines (IgG, $\operatorname{IgM})$ were increased at the $2^{\text {nd }}$ week of age followed by significant decrease by the protective effects of Nigella. On the other hand, IgE was increased and maintained at high levels. A sharp decline in both Total Bacterial Count and Total Enterobacteriaceae Count until the $4^{\text {th }}$ week of age followed by sharp increase in both types in all treated groups suggesting the failure of the protective action. A follow up with the supplementation in higher doses may protect from the further changes in biochemical, antioxidant, immunological and bacteriological aspects.
\end{abstract}

Copy Right, IJAR, 2017,. All rights reserved.

\section{Introduction:-}

Nigella sativa was known in traditional and religious medicine for almost centuries in India, China, Far East and Middle East as a natural treatment for many diseases and as a flavoring agent that claimed a medicinal usage traced back to the ancient Egyptians, Greeks, and Romans; Elkhayat et al., (2016). Nigella seeds have many medicinal actions such as bronchodilator, hypotensive, antibacterial, antifungal, anti-diabetic, anticancer; Periasamy et al. (2016), analgesic, anti-inflammatory, antidepressant; Sahak et al., (2016) and immune-potentiating actions.

Corresponding Author:- Essam S Soliman.

Address:- Department of Animal Hygiene, Zoonosis \& Animal Behavior, Faculty of Veterinary Medicine, Suez Canal University, Ismailia, Egypt, 41522. 
Nigella was able chemically to reduce the levels of triglycerides and glucose once they were elevated in the body, as well as can stabilize cholesterol metabolism. Despite of its large benefits in the medicinal field, Nigella seeds were neglected and left behind although, Nigella sativa may possibly induce P-glycoprotein activity, Hammad Shafiq et al., (2014).

Diazinon is one of the most commonly used organophosphorus compounds; and have many applications in the veterinary and agricultural fields; Ghoneim et al., (2001). Diazinon is an organic phosphate and cholinesterase enzyme inhibitor that commonly used for the control of common chicken lice and other variety of insect in poultry farms. Diazinon was not recommended to be used in the presence of a live bird as chickens will consume the diazinon crystals, results in lacrimation, diarrhea, dyspnea, and death. Postmortem finding from toxicity may include lung edema, fatty livers, severe enteritis and the inclusion of diazinon crystals in the crop and gizzard contents.

The present study aimed for evaluating the influences of supplementary different concentration of Nigella sativa on antioxidant activity [superoxide dismutase (SOD), glutathione peroxidase (GSH-Px) and glutathione reductase], blood chemistry, bacterial load and immunological status before and after toxifying birds with synthetized diazinon.

\section{Material and methods:-}

Experimental birds and study design:-

150 broilers (one day old chicks) were purchased and placed under the same environmental conditions for acclimatization. The birds were divided into 3 groups (G1, G2, and control G3) according to the supplemented concentration of Nigella sativa. G1 supplemented with 1.4\% Nigella sativa (1.4 gm / 100 gm ration); G2 supplemented with 2.8\% Nigella sativa (2.8 gm / 100 gm ration) and G3 was kept as control. G1 and G2 were toxified with synthetized diazinon (100 ppm / $1 \mathrm{~L}$ drinking water; LD50=6.31 mg $/ \mathrm{kg}$ ) after it have been compared to the commercial form for its chemical and physical properties as well as the compound stability.

\section{Diazinon:-}

Diazinon was synthetized in the laboratory and the reaction mixture was then washed first with $400 \mathrm{~mL}$ of $0.1 \mathrm{~N}$ hydrochloric acid and then with $400 \mathrm{~mL}$ of $0.1 \mathrm{~N}$ sodium hydroxide solution. After Separating off the washing liquids, the xylene was distilled off in vacuum for the complete removal of the volatile constituents, the diazinon was finally distilled in a high vacuum (approximately $1 \mathrm{mbar}$ ) at $60{ }^{\circ} \mathrm{C}$. The yield of 0,0 diethyl $-0-(2-$ isopropyl $-4-$ methyl -6 - pyrimidinyl) the ionophosphate was $280 \mathrm{~g}=92.1 \%$ of the theoretical yield, Budavari, (1996); Hansch et al., (1995); Geller et al., (2003).

\section{Sampling and samples preparation:-}

Blood samples: A total of 180 samples were collected during the study period, these samples were collected on a weekly basis starting from $14^{\text {th }}$ day old (zero sample), and three post challenge sample collections (P1, P2, and P3). Serum blood samples (60 samples) were left in a cool place for $2-3 \mathrm{hrs}$, then centrifuged at $3000 \mathrm{rpm} / 5 \mathrm{~min}$. The serum samples were separated in Eppendorf tube of $3 \mathrm{~mL}$ capacity and kept frozen until examination for Blood biochemical parameters as Total Protein (TP); Albumin (ALB); Globulin (Glob); liver enzymes as alanine aminotransferase (ALT); aspartate aminotransferase (AST); Cholesterol (CHOL); Triglycerides (TG); Urea (Ure); Creatinine and Glucose (Gluc), the parameters were measured calometerically using UV1100 spectrophotometer; Young, (1995-2001), immunological assay was also performed on serum samples (IgG, IgM, IgE) using DIFFU plate method; Berne, (1974), Heremans and Masson, (1973). The whole blood samples (60 samples) were centrifuged at $4000 \mathrm{rpm} / 10 \mathrm{~min}$; then aspirate off the plasma and stored at $-80{ }^{\circ} \mathrm{C}$. The erythrocytes were washed 34 times with $5 \mathrm{~mL}$ sodium chloride $0.9 \%$ solution and centrifuged after each wash at $4000 \mathrm{rpm} / 10 \mathrm{~min}$. Finally the washed erythrocytes were re-suspended in cold distilled water and mixed; the lysate were stored at $-80{ }^{\circ} \mathrm{C}$ for antioxidant assay as superoxide dismutase; SOD (Nishikimi et al., 1972), glutathione peroxidase; GSH-Px (Paglia and Valentine, 1967). and glutathione reductase (Goldberg and Spooner, 1983).

Intestinal swab (60 samples); from the original dilution of all swab sample, $1 \mathrm{~mL}$ was transferred aseptically to a test tube containing $9 \mathrm{~mL}$ sterile $0.1 \%$ buffered peptone water $(\mathrm{w} / \mathrm{v})$ to prepare a dilution of $10^{-2}$, then from which tenfold decimal serial dilution up to $10^{-6}$ were prepared to cover a wide range of samples contamination which could be easily counted. The dilutions were subjected to two types of bacterial counts; Total Bacterial Count (TBC) and Total Enterobacterieacae Count (TEC). 


\begin{abstract}
Bacterial Counts:-
Total Bacterial Count (TBC); The number of Aerobic spore forming micro-organisms in samples was carried out using drop Plate Method by Zelver et al., (1999) and Herigstad et al., (2001)using standard plat count agar. The inoculated and un-inoculated control plates were incubated at $37{ }^{\circ} \mathrm{C}$ for $24-48$ hours. Total Enterobacterieacae Count (TEC); was performed using drop Plate Method; Zelver et al., (1999) and Herigstad et al., (2001) with Eosine Methylene Blue Agar (EMB).Plates were inverted and incubated at $37{ }^{\circ} \mathrm{C}$ for $24-48$ hours. Counting the colonies on the plates showed 30-300 colonies per plates; Cruickshank et al., (1975, 1980). Five typical colonies were selected and cultured onto MacConkey agar plates, and incubated at $37{ }^{\circ} \mathrm{C}$ for $24 \mathrm{hr}$, the pure colonies on MacConkey agar plates were inoculated onto nutrient slant and incubated at $37{ }^{\circ} \mathrm{C}$ for $24 \mathrm{hr}$ and kept for further identification.
\end{abstract}

\title{
Statistical analysis:-
}

Statistical analysis was run through SPSS (version, 16) for windows Levesque, (2007). The obtained data were analyzed statistically using factorial experiments of Analysis of Variance (ANOVA) with general linear model procedures (GLM) for all tested groups, times and their interactions.

\section{Results:-}

Proteinogram in Table 1 revealed that total protein, Albumin and Globulin revealed non-significant $(\mathrm{P} \leq 0.05)$ differences between G1, G2 and control at different sampling times. As well as, ALT, AST (Table 2) showed nonsignificant differences $(\mathrm{P} \leq 0.05)$ in all groups at all sampling times. Urea (Table 2) revealed a non-significant increase $(\mathrm{P}<0.05)$ in $\mathrm{G} 1$ and $\mathrm{G} 2$ compare to control at $\mathrm{P} 1, \mathrm{P} 2$ and a non-significant decrease $(\mathrm{P}<0.05)$ in G1 and G2 compare to control at P3. Creatinine revealed significant decline $(\mathrm{P} \leq 0.05)$ in $\mathrm{G} 2$ and control compared to G1 at zero time and a highly significant decrease $(\mathrm{P}<0.01)$ in control compared to $\mathrm{G} 1$ and $\mathrm{G} 2$ at $\mathrm{P} 1$ sampling time. Meanwhile, at $\mathrm{P} 2$ and $\mathrm{P} 3$ there was a non-significant difference $(\mathrm{P}>0.05)$ between groups.

Triglycerides and cholesterol (Table 3 ) showed a highly significant decrease $(\mathrm{P}<0.01)$ in G1 and G2 compared to control at zero sampling time and a non-significant difference $(\mathrm{P}>0.05)$ between groups at $\mathrm{P} 1, \mathrm{P} 2$ and $\mathrm{P} 3$ sampling times. Glucose showed a highly significant decrease $(\mathrm{P}<0.01)$ in $\mathrm{G} 1$ and $\mathrm{G} 2$ compared to control group at $\mathrm{P} 1$ and significant decrease $(\mathrm{P} \leq 0.05)$ in $\mathrm{G} 1$ and control groups compared to $\mathrm{G} 1$ at $\mathrm{P} 2$ sampling time.

Glutathione peroxidase (Table 4) showed a highly significant $(\mathrm{P}<0.01)$ decrease in $\mathrm{G} 2$ and control groups compared to $\mathrm{G} 1$ at zero and $\mathrm{P} 1$ sampling times, and a non-significant difference $(\mathrm{P}>0.05)$ between all groups at $\mathrm{P} 2$ and $\mathrm{P} 3$ sampling times. Glutathione reductase and Superoxide dimutase revealed significant difference $(\mathrm{P} \leq 0.05)$ between groups at zero and P2 sampling times.

Immunoglobines $(\mathrm{IgG})$ revealed a highly significant difference $(\mathrm{P}<0.01)$ in Table $(5)$ between groups during all sampling times and non-significant $(\mathrm{P}>0.05)$ decrease between $\mathrm{G} 2$ and control compared to $\mathrm{G} 1$ at $\mathrm{P} 1$ sampling time. IgM profile showed a highly significant difference $(\mathrm{P}<0.01)$ between groups at all sampling times; a non-significant decrease $(\mathrm{P}>0.05)$ between $\mathrm{G} 2$ and control compared to $\mathrm{G} 1$ at zero sampling time; a non-significant decrease $(\mathrm{P}>0.05)$ between G1 and G2 compared to control at P2 sampling time and a non-significant difference $(\mathrm{P}>0.05)$ between G1 and control at P3 sampling time; Table (5). IgE revealed a highly significant difference $(\mathrm{P}<0.01)$ between groups at zero and P1 sampling times, and a non-significant decrease (P>0.05) in G1 and G2 compared to control at the same sampling time. A significant difference $(\mathrm{P} \leq 0.05)$ at $\mathrm{P} 2$ and non-significant difference $(\mathrm{P}>0.05)$ at P3 sampling time, Table (5).

TBC revealed in Table 5 a highly significant $(\mathrm{P}<0.01)$ difference between all groups at all sampling times, and a non-significant decrease $(\mathrm{P}>0.05)$ between G2 and control compared to G1 at P2 sampling time. Meanwhile, TEC showed a highly significant difference $(\mathrm{P}>0.01)$ between all groups at all sampling times and non-significant (P>0.05) difference between G1 and control; G1 and G2; G2 and control and G2 and control at zero, P1, P2, and P3 sampling times; respectively.

$\operatorname{IgM}$ and IgE (Table 6) showed a highly significant strong positive correlation $(\mathrm{r}=0.646 ; \mathrm{P}<0.01)$ with $\mathrm{TBC}$. Table (7) revealed a significant weak negative correlation $(r=-0.394 ; \mathrm{P} \leq 0.05)$ between $\mathrm{TBC}$ and Superoxide Dimutase. TEC in Table (7) showed a significant weak negative correlation with SOD ( $r=-0.336 ; \mathrm{P} \leq 0.05)$. 


\section{Discussion:-}

A variety of medicinal plants as well as their extracted and purified constituents have shown many therapeutic effects. On a wide spectrum, medicinal plants was able to serve as therapeutic, medicinal alternatives, safer choices and effective treatment. Animals, broilers and human-being in a variety of cultures can be treated or prophylactic with medicinal plants. Nigella sativa from family Ranunculaceae, have been used for centuries as food preservative and medicinal plant. The oil constituents; thymoquinine (TQ), have shown a potential medicinal properties, as it possess anti-oxidant effects by enhancing the oxidant scavenger system, which as a consequence causes antitoxic effects induced by several problems; Salem, (2005). The present study showed that Glutathione peroxidase possess a highly significant decrease in G2 and control groups compared to G1 at zero and P1 sampling times, and a nonsignificant difference between all groups at P2 and P3 sampling times. Glutathione reductase and Superoxide dimutase revealed significant difference between groups at zero and P2 sampling times.

Several diseases serve to contribute oxidative damage to many biological structures during their pathophysiological course especially in cardiovascular diseases and cancer. The oxidative damage was caused by imbalance between the pro-oxidant (free radicals) and the anti-oxidant (scavenging) mediators, as pro-oxidant predominated either by the increased generation of the free radicals contributed by excessive oxidative stress conditions, or by the poor scavenging capability in the body. Free oxygen radicals; $\mathrm{O}_{2}, \mathrm{OH}^{-}$, and $\mathrm{NO}^{-}$that are developed during a stress condition; are electrically charged and attack cells causing tearing of the cellular membranes and created a havoc with the nucleic acids, proteins, and enzymes existed in animal or human body, causing the cell to lose its structure and function completely. ROS are produced mainly by certain cells of immune system as macro-phages and neutrophils;

Maxwell, (1999); Schulz et al., (2000); Hogg, (1998).

The present study showed a sharp increase in the lipid profile and glucose, and this was attributed to diazinon intoxication. The elevated levels was declined latter as the bird proceed in age and this was attributed to the ability Nigella sativa to decrease the lipid peroxidation, increase the anti-oxidant mechanism and prevent the lipidperoxidation-induced liver damage. $N$. sativa also was able to decrease the elevated glucose and MDA concentrations, increased the lowered GSH and ceruloplasmin concentrations. These results were supported with the finding from experimentally infected rabbits in Meral et al., (2001); Mariod et al., (2009), and Umar et al., (2012).

Nigella sativa showed beneficial immunomodulatory properties, as it was able to stimulate T cell- and natural killer cell-mediated immune responses. The oil and its active ingredients proved an excellent anti-microbial and antitumor properties. Gathering these beneficial and medicinal influences, N. sativa seed is a promising source for active ingredients that would be with potential therapeutic modalities in different clinical settings; Salem, (2005).Immunoglobines ( $\operatorname{IgG}, \operatorname{IgM})$ in the present study revealed a highly significant difference between groups during all sampling times with potential increase followed by decrease from the $3^{\text {rd }}$ week of age proving the presence of immune barrier, that did not last for a period of time.

Table 1. Biochemical changes in proteinogram by groups supplemented with different Nigella sativa concentrations against Diazinon (OPC) toxicity.

\begin{tabular}{|c|c|c|c|c|}
\hline Time & Group & $\begin{array}{c}\text { Total Protein } \\
\text { g/dl } \\
\end{array}$ & $\begin{array}{c}\text { Albumin } \\
\text { g/dl }\end{array}$ & $\begin{array}{c}\text { Globulin } \\
\text { g/dl }\end{array}$ \\
\hline \multirow{3}{*}{$\stackrel{0}{\stackrel{0}{j}}$} & G1 & $7.373 \pm 2.445^{\mathrm{a}}$ & $1.828 \pm 0.242^{\mathrm{a}}$ & $5.546 \pm 2.653^{\mathrm{a}}$ \\
\hline & G2 & $9.181 \pm 5.032^{\mathrm{a}}$ & $1.054 \pm 0.385^{\mathrm{a}}$ & $8.127 \pm 4.650^{\mathrm{a}}$ \\
\hline & G3 & $18.048 \pm 4.347^{\mathrm{a}}$ & $0.887 \pm 0.489^{\mathrm{a}}$ & $17.161 \pm 3.927^{\mathrm{a}}$ \\
\hline \multirow{3}{*}{$\bar{a}$} & G1 & $39.181 \pm 10.161^{\mathrm{a}}$ & $2.161 \pm 0.088^{\mathrm{a}}$ & $37.020 \pm 10.077^{\mathrm{a}}$ \\
\hline & G2 & $35.108 \pm 10.282^{\mathrm{a}}$ & $2.164 \pm 0.702^{\mathrm{a}}$ & $32.945 \pm 9.585^{\mathrm{a}}$ \\
\hline & G3 & $25.687 \pm 11.704^{\mathrm{a}}$ & $0.754 \pm 0.154^{\mathrm{a}}$ & $24.933 \pm 11.858^{\mathrm{a}}$ \\
\hline \multirow{3}{*}{$\mathfrak{\Sigma}$} & G1 & $32.048 \pm 9.338^{\mathrm{a}}$ & $1.805 \pm 0.199^{\mathrm{a}}$ & $30.243 \pm 9.537^{\mathrm{a}}$ \\
\hline & G2 & $29.157 \pm 1.193^{\mathrm{a}}$ & $1.867 \pm 0.500^{\mathrm{a}}$ & $27.289 \pm 0.997^{\mathrm{a}}$ \\
\hline & G3 & $32.024 \pm 3.842^{\mathrm{a}}$ & $1.573 \pm 0.225^{\mathrm{a}}$ & $30.451 \pm 3.681^{\mathrm{a}}$ \\
\hline \multirow{3}{*}{$\tilde{\varepsilon}$} & G1 & $26.675 \pm 8.058^{\mathrm{a}}$ & $1.763 \pm 0.225^{\mathrm{a}}$ & $24.912 \pm 8.216^{\mathrm{a}}$ \\
\hline & G2 & $24.988 \pm 3.463^{\mathrm{a}}$ & $1.218 \pm 0.057^{\mathrm{a}}$ & $23.770 \pm 3.517^{\mathrm{a}}$ \\
\hline & G3 & $26.434 \pm 6.637^{\mathrm{a}}$ & $1.418 \pm 0.270^{\mathrm{a}}$ & $25.016 \pm 6.368^{\mathrm{a}}$ \\
\hline
\end{tabular}


G1 Broilers supplemented with 1.4\% Nigella sativa

G2 Broilers supplemented with $2.8 \%$ Nigella sativa

G3 control

Means carrying different superscripts in the same column are significantly different at $(\mathrm{P} \leq 0.05)$ or highly significantly different at $(\mathrm{P}<0.01)$.

Means carrying the same superscripts in the same column are non-significantly different at $(\mathrm{P}>0.05)$.

Table 2. Biochemical changes in liver \& kidney function by groups supplemented with different Nigella sativa concentrations against Diazinon (OPC) toxicity.

\begin{tabular}{|c|c|c|c|c|c|}
\hline Time & Group & $\begin{array}{l}\text { ALT } \\
\text { U / L }\end{array}$ & $\begin{array}{c}\text { AST } \\
\text { U / L }\end{array}$ & $\begin{array}{l}\text { Urea } \\
\text { mg / dl }\end{array}$ & $\begin{array}{c}\text { Creatinine } \\
\text { mg / dl }\end{array}$ \\
\hline \multirow{3}{*}{ 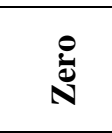 } & G1 & $3.200 \pm 1.124^{\mathrm{a}}$ & $3.733 \pm 0.309^{\mathrm{a}}$ & $18.052 \pm 0.567^{\mathrm{a}}$ & $2.057 \pm 0.313^{\mathrm{a}}$ \\
\hline & G2 & $1.653 \pm 0.107^{\mathrm{a}}$ & $1.750 \pm 0.926^{\mathrm{a}}$ & $23.095 \pm 0.677^{\mathrm{a}}$ & $0.766 \pm 0.154^{b}$ \\
\hline & G3 & $1.493 \pm 0.232^{\mathrm{a}}$ & $1.867 \pm 0.617^{\mathrm{a}}$ & $25.982 \pm 5.404^{\mathrm{a}}$ & $1.117 \pm 0.360^{\mathrm{ab}}$ \\
\hline \multirow{3}{*}{$\bar{a}$} & G1 & $0.853 \pm 0.053^{\mathrm{a}}$ & $9.450 \pm 4.554^{\mathrm{a}}$ & $23.364 \pm 0.828^{\mathrm{a}}$ & $0.951 \pm 0.037^{\mathrm{a}}$ \\
\hline & G2 & $0.800 \pm 0.562^{\mathrm{a}}$ & $5.133 \pm 3.739^{\mathrm{a}}$ & $19.361 \pm 1.544^{\mathrm{a}}$ & $0.412 \pm 0.102^{b}$ \\
\hline & G3 & $0.800 \pm 0.640^{\mathrm{a}}$ & $3.967 \pm 3.106^{\mathrm{a}}$ & $8.545 \pm 5.079^{b}$ & $0.341 \pm 0.156^{\mathrm{b}}$ \\
\hline \multirow{3}{*}{$\Sigma$} & G1 & $1.547 \pm 0.282^{\mathrm{a}}$ & $9.217 \pm 2.510^{\mathrm{a}}$ & $19.900 \pm 0.491^{\mathrm{a}}$ & $0.484 \pm 0.039^{\mathrm{a}}$ \\
\hline & G2 & $5.013 \pm 2.399^{\mathrm{a}}$ & $5.017 \pm 3.365^{\mathrm{a}}$ & $12.933 \pm 5.543^{\mathrm{a}}$ & $0.362 \pm 0.237^{\mathrm{a}}$ \\
\hline & G3 & $4.587 \pm 2.356^{\mathrm{a}}$ & $5.367 \pm 3.285^{\mathrm{a}}$ & $13.818 \pm 6.034^{\mathrm{a}}$ & $0.823 \pm 0.365^{\mathrm{a}}$ \\
\hline \multirow{3}{*}{$\tilde{2}$} & G1 & $1.600 \pm 0.277^{\mathrm{a}}$ & $5.950 \pm 2.865^{\mathrm{a}}$ & $18.938 \pm 1.447^{\mathrm{a}}$ & $0.973 \pm 0.504^{\mathrm{a}}$ \\
\hline & G2 & $1.547 \pm 0.533^{\mathrm{a}}$ & $4.200 \pm 3.676^{\mathrm{a}}$ & $20.131 \pm 1.485^{\mathrm{a}}$ & $1.048 \pm 0.435^{\mathrm{a}}$ \\
\hline & G3 & $2.507 \pm 1.102^{\mathrm{a}}$ & $2.683 \pm 1.184^{\mathrm{a}}$ & $28.907 \pm 5.530^{\mathrm{a}}$ & $0.908 \pm 0.417^{\mathrm{a}}$ \\
\hline \multicolumn{6}{|c|}{$\begin{array}{l}\text { G1 Broilers supplemented with } 1.4 \% \text { Nigella sativa } \\
\text { G2 Broilers supplemented with } 2.8 \% \text { Nigella sativa } \\
\text { G3 control } \\
\text { Means carrying different superscripts in the same column are significantly different at }(\mathrm{P} \leq 0.05) \text { or highly } \\
\text { significantly different at }(\mathrm{P}<0.01) \text {. } \\
\text { Means carrying the same superscripts in the same column are non-significantly different at }(\mathrm{P}>0.05) \text {. }\end{array}$} \\
\hline
\end{tabular}

Table 3. Biochemical changes in lipid profile \& glucose function by groups supplemented with different Nigella sativa concentrations against Diazinon (OPC) toxicity.

\begin{tabular}{|c|c|c|c|c|}
\hline Time & Group & $\begin{array}{c}\text { Triglycerides } \\
\text { mg / dl }\end{array}$ & $\begin{array}{c}\text { Cholesterol } \\
\text { mg / dl }\end{array}$ & $\begin{array}{c}\text { Glucose } \\
\text { mg / dl }\end{array}$ \\
\hline \multirow{3}{*}{ 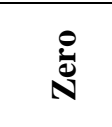 } & G1 & $87.255 \pm 22.162^{b}$ & $26.667 \pm 1.633^{b}$ & $882.143 \pm 43.055^{\mathrm{a}}$ \\
\hline & G2 & $146.078 \pm 21.502^{b}$ & $81.569 \pm 37.650^{b}$ & $990.476 \pm 55.380^{\mathrm{a}}$ \\
\hline & G3 & $378.431 \pm 43.965^{\mathrm{a}}$ & $236.340 \pm 18.573^{\mathrm{a}}$ & $915.476 \pm 124.750^{\mathrm{a}}$ \\
\hline \multirow{3}{*}{$\bar{E}$} & G1 & $533.333 \pm 163.866^{\mathrm{a}}$ & $184.052 \pm 55.219^{\mathrm{a}}$ & $1060.714 \pm 49.616^{b}$ \\
\hline & G2 & $209.804 \pm 4.273^{\mathrm{a}}$ & $130.980 \pm 32.236^{\mathrm{a}}$ & $1141.667 \pm 111.161^{b}$ \\
\hline & G3 & $187.255 \pm 47.191^{\mathrm{a}}$ & $191.373 \pm 59.268^{\mathrm{a}}$ & $1505.952 \pm 45.612^{\mathrm{a}}$ \\
\hline \multirow{3}{*}{$\tilde{\Sigma}$} & G1 & $730.392 \pm 535.101^{\mathrm{a}}$ & $327.843 \pm 17.544^{\mathrm{a}}$ & $182.143 \pm 98.263^{b}$ \\
\hline & G2 & $296.078 \pm 45.953^{\mathrm{a}}$ & $260.131 \pm 66.850^{\mathrm{a}}$ & $1192.857 \pm 154.812^{\mathrm{a}}$ \\
\hline & G3 & $408.824 \pm 109.313^{\mathrm{a}}$ & $212.549 \pm 104.265^{\mathrm{a}}$ & $783.333 \pm 281.172^{\mathrm{ab}}$ \\
\hline \multirow{3}{*}{$\stackrel{n}{=}$} & G1 & $767.647 \pm 518.602^{\mathrm{a}}$ & $321.830 \pm 11.680^{\mathrm{a}}$ & $291.667 \pm 101.526^{\mathrm{a}}$ \\
\hline & G2 & $343.137 \pm 57.242^{\mathrm{a}}$ & $149.804 \pm 62.796^{\mathrm{a}}$ & $780.952 \pm 275.535^{\mathrm{a}}$ \\
\hline & G3 & $381.373 \pm 118.688^{\mathrm{a}}$ & $303.007 \pm 82.010^{\mathrm{a}}$ & $1016.667 \pm 503.872^{\mathrm{a}}$ \\
\hline \multicolumn{5}{|c|}{$\begin{array}{l}\text { G1 Broilers supplemented with } 1.4 \% \text { Nigella sativa } \\
\text { G2 Broilers supplemented with } 2.8 \% \text { Nigella sativa } \\
\text { G3 control } \\
\text { Means carrying different superscripts in the same column are significantly different at }(\mathrm{P} \leq 0.05) \text { or highly } \\
\text { significantly different at }(\mathrm{P}<0.01) \text {. } \\
\text { Means carrying the same superscripts in the same column are non-significantly different at }(\mathrm{P}>0.05) \text {. }\end{array}$} \\
\hline
\end{tabular}


Table 4. Antioxidant enzymatic changes by groups supplemented with different Nigella sativa concentrations against Diazinon (OPC) toxicity.

\begin{tabular}{|c|c|c|c|c|}
\hline Time & Group & $\begin{array}{l}\text { GSH-Px } \\
\text { mU / mL }\end{array}$ & $\begin{array}{c}\text { GSH-Rx } \\
\text { U / L }\end{array}$ & $\begin{array}{c}\text { SOD } \\
\mathbf{U} / \mathbf{m L}\end{array}$ \\
\hline \multirow{3}{*}{ 总 } & G1 & $7943.462 \pm 1685.959^{\mathrm{a}}$ & $475.582 \pm 415.297^{b}$ & $2817.118 \pm 1154.865^{b}$ \\
\hline & G2 & $2042.604 \pm 38.907^{b}$ & $1714.773 \pm 328.218^{\mathrm{ab}}$ & $6357.759 \pm 563.306^{\mathrm{a}}$ \\
\hline & G3 & $1465.488 \pm 85.043^{b}$ & $3215.200 \pm 541.572^{\mathrm{a}}$ & $4418.103 \pm 731.341^{\mathrm{ab}}$ \\
\hline \multirow{3}{*}{$\bar{a}$} & G1 & $5362.647 \pm 590.086^{\mathrm{a}}$ & $2411.400 \pm 1004.750^{\mathrm{a}}$ & $7635.468 \pm 2017.330^{\mathrm{a}}$ \\
\hline & G2 & $3423.794 \pm 291.801^{b}$ & $1976.008 \pm 1935.818^{\mathrm{a}}$ & $4602.833 \pm 173.482^{\mathrm{a}}$ \\
\hline & G3 & $1990.729 \pm 244.611^{\mathrm{c}}$ & $864.085 \pm 90.613^{\mathrm{a}}$ & $6834.975 \pm 3214.343^{\mathrm{a}}$ \\
\hline \multirow{3}{*}{$\tilde{\Sigma}$} & G1 & $635.477 \pm 103.751^{\mathrm{a}}$ & $569.358 \pm 448.788^{b}$ & $6434.729 \pm 1534.552^{\mathrm{ab}}$ \\
\hline & G2 & $1841.586 \pm 745.713^{\mathrm{a}}$ & $7890.637 \pm 113.872^{\mathrm{a}}$ & $8097.290 \pm 203.645^{\mathrm{a}}$ \\
\hline & G3 & $1660.021 \pm 1195.042^{\mathrm{a}}$ & $3436.245 \pm 2346.301^{\mathrm{ab}}$ & $2478.449 \pm 1522.926^{b}$ \\
\hline \multirow{3}{*}{$\tilde{\theta}$} & G1 & $1614.630 \pm 622.508^{\mathrm{a}}$ & $3262.088 \pm 529.168^{\mathrm{a}}$ & $1631.773 \pm 226.769^{\mathrm{a}}$ \\
\hline & G2 & $1018.060 \pm 640.322^{\mathrm{a}}$ & $4903.180 \pm 535.825^{\mathrm{a}}$ & $1862.685 \pm 333.381^{\mathrm{a}}$ \\
\hline & G3 & $486.334 \pm 198.704^{\mathrm{a}}$ & $3262.088 \pm 487.462^{\mathrm{a}}$ & $2924.877 \pm 512.417^{\mathrm{a}}$ \\
\hline \multicolumn{5}{|c|}{$\begin{array}{l}\text { G1 Broilers supplemented with } 1.4 \% \text { Nigella sativa } \\
\text { G2 Broilers supplemented with } 2.8 \% \text { Nigella sativa } \\
\text { G3 control } \\
\text { Means carrying different superscripts in the same column are significantly different at }(\mathrm{P} \leq 0.05) \text { or highly } \\
\text { significantly different at }(\mathrm{P}<0.01) \text {. } \\
\text { Means carrying the same superscripts in the same column are non-significantly different at }(\mathrm{P}>0.05) \text {. }\end{array}$} \\
\hline
\end{tabular}

Table 5. Immunological and bacteriological changes by groups supplemented with different Nigella sativa concentrations against Diazinon (OPC) toxicity.

\begin{tabular}{|c|c|c|c|c|c|c|}
\hline Time & Group & $\begin{array}{c}\text { IgG } \\
\mathrm{mg} / \mathrm{dl}\end{array}$ & $\begin{array}{c}\text { IgM } \\
\mathrm{mg} / \mathrm{dl}\end{array}$ & $\begin{array}{c}\text { IgE } \\
\text { IU / mL }\end{array}$ & $\begin{array}{c}\text { TBC } \\
\text { CFU / mL }\end{array}$ & $\begin{array}{c}\text { TEC } \\
\text { CFU / mL }\end{array}$ \\
\hline \multirow{3}{*}{ 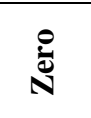 } & G1 & $8.433 \pm 0.384^{\mathrm{a}}$ & $9.900 \pm 0.058^{\mathrm{a}}$ & $6.667 \pm 0.219^{b}$ & $13.8 \times 10^{3} \pm 600.925^{b}$ & $1.4 \times 10^{1} \pm 2.404^{b}$ \\
\hline & G2 & $6.100 \pm 0.058^{b}$ & $4.067 \pm 0.067^{b}$ & $6.467 \pm 0.176$ & $16.2 \times 10^{3} \pm 635.959^{a}$ & $1.1 \times 10^{2} \pm 7.638^{\mathrm{a}}$ \\
\hline & G3 & $3.867 \pm 0.949^{c}$ & $4.500 \pm 0.500^{\mathrm{b}}$ & $10.667 \pm 0.667^{\mathrm{a}}$ & $4.5 \times 10^{3} \pm 404.145^{\mathrm{c}}$ & $1.4 \times 10^{1} \pm 2.333^{b}$ \\
\hline \multirow{3}{*}{ E } & G1 & $21.100 \pm 2.098^{\mathrm{a}}$ & $12.133 \pm 0.353^{b}$ & $8.800 \pm 0.208^{b}$ & $3.4 \times 10^{3} \pm 338.296^{\mathrm{a}}$ & $5.8 \times 10^{1} \pm 6.009^{\mathrm{a}}$ \\
\hline & G2 & $8.567 \pm 0.384^{b}$ & $5.800 \pm 0.404^{\mathrm{c}}$ & $7.467 \pm 0.06^{\mathrm{b}}$ & $3.1 \times 10^{2} \pm 12.019^{c}$ & $4 \times 10^{1} \pm 8.083^{\mathrm{a}}$ \\
\hline & G3 & $4.833 \pm 0.833^{b}$ & $19.667 \pm 1.856^{\mathrm{a}}$ & $28.400 \pm 6.032^{\mathrm{a}}$ & $1.3 \times 10^{3} \pm 28.868^{b}$ & $0.0 \pm 0.000^{b}$ \\
\hline \multirow{3}{*}{$\Sigma$} & G1 & $5.367 \pm 0.470^{b}$ & $3.233 \pm 0.186^{b}$ & $13.600 \pm 2.346^{\mathrm{ab}}$ & $2.4 \times 10^{2} \pm 38.442^{\mathrm{a}}$ & $7.5 \times 10^{2} \pm 31.798^{\mathrm{a}}$ \\
\hline & G2 & $4.033 \pm 0.033^{\mathrm{c}}$ & $2.233 \pm 0.186^{b}$ & $8.033 \pm 0.786^{b}$ & $3.5 \times 10^{1} \pm 3.180^{b}$ & $0.0 \pm 0.000^{b}$ \\
\hline & G3 & $8.933 \pm 0.470^{\mathrm{a}}$ & $24.667 \pm 1.453^{\mathrm{a}}$ & $17.700 \pm 1.762^{\mathrm{a}}$ & $4.3 \times 10^{3} \pm 1.856^{b}$ & $0.0 \pm 0.000^{\mathrm{b}}$ \\
\hline \multirow{3}{*}{ 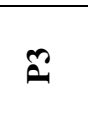 } & G1 & $8.767 \pm 0.384^{\mathrm{a}}$ & $5.467 \pm 0.418^{\mathrm{a}}$ & $9.400 \pm 0.643^{\mathrm{a}}$ & $22 \times 10^{3} \pm 1527.525^{b}$ & $12.3 \times 10^{3} \pm 1452.966^{\mathrm{a}}$ \\
\hline & G2 & $7.233 \pm 0.722^{\mathrm{a}}$ & $3.733 \pm 0.504^{b}$ & $10.833 \pm 1.837^{\mathrm{a}}$ & $41 \times 10^{3} \pm 577.350^{\mathrm{a}}$ & $1.7 \times 10^{3} \pm 378.594^{b}$ \\
\hline & G3 & $4.900 \pm 0.802^{\mathrm{b}}$ & $6.167 \pm 0.088^{\mathrm{a}}$ & $13.567 \pm 1.065^{\mathrm{a}}$ & $9.3 \times 10^{2} \pm 8.819^{c}$ & $1.7 \times 10^{3} \pm 44.096^{b}$ \\
\hline \multicolumn{7}{|c|}{$\begin{array}{l}\text { G1 Broilers supplemented with } 1.4 \% \text { Nigella sativa } \\
\text { G2 Broilers supplemented with } 2.8 \% \text { Nigella sativa } \\
\text { G3 control } \\
\text { Means carrying different superscripts in the same column are significantly different at }(\mathrm{P} \leq 0.05) \text { or highly significantly } \\
\text { different at }(\mathrm{P}<0.01) \text {. } \\
\text { Means carrying the same superscripts in the same column are non-significantly different at }(\mathrm{P}>0.05) \text {. }\end{array}$} \\
\hline
\end{tabular}

Table 6. Correlation co-efficient of immunoglobines with Total Bacterial Count (TBC) Above Diagonal and Total Enterobacteracaea Count (TEC) Below Diagonal.

\begin{tabular}{|c|c|c|c|c|}
\hline $\mathbf{r}$ & TBC & IgG & IgM & IgE \\
\hline TEC & $\mathbf{1}$ & 0.24 & -0.304 & -0.248 \\
\hline IgG & 0.043 & $\mathbf{1}$ & 0.278 & -0.158 \\
\hline IgM & -0.185 & 0.278 & $\mathbf{1}$ & 0.646 \\
\hline IgE & -0.096 & -0.158 & 0.646 & $\mathbf{1}$ \\
\hline **. Correlation is highly significant $(\mathrm{P}<0.01)$. & $*$. Correlation is significant $(\mathrm{P}<0.05)$. \\
Ns. Correlation is non-significant $(\mathrm{P}>0.05)$.
\end{tabular}


Table 7. Correlation co-efficient of antioxidants with Total Bacterial Count (TBC) Above Diagonal and Total Enterobacteracaea Count (TEC) Below Diagonal.

\begin{tabular}{|c|c|c|c|c|}
\hline $\mathbf{r}$ & TBC & GSH-Px & GSH-Rx & SOD \\
\hline TEC & 1 & -0.015 & 0.114 & -0.394 \\
\hline GSH-Px & -0.191 & 1 & -0.327 & 0.136 \\
\hline GSH-Rx & 0.074 & -0.327 & 1 & 0.021 \\
\hline SOD & -0.366 & 0.136 & 0.021 & 1 \\
\hline
\end{tabular}

\section{Conclusion:-}

Nigella sativa supplementations $(1.4 \%, 2.8 \%)$ were able to protect the birds from intoxication at once up to the $4^{\text {th }}$ week of age. Although it maintained the biochemical parameters in a normal levels after little deviation resulted from intoxication. Antioxidants were severely stimulated by the Nigella sativa supplementations $(1.4 \%, 2.8 \%)$ to initiate its protective effect. A follow up with the supplementation in higher doses may protect from the further changes in biochemical, antioxidant, immunological and bacteriological aspects.

\section{References:-}

1. Berne, G.H. (1974). Clin. Chem. 200: 61-89.

2. Budavari, S. ed. (1996). The Merck Index - An Encyclopedia of Chemicals, Drugs, and Biologicals. Whitehouse Station, NJ: Merck. p. 508.

3. Cruickshank, R., Duguid, J.P., Marimion, B.P., and Swain, R.H. (1975). Medical Microbiology, the Practice of Medical Microbiology. 12th Ed, Vol. 11, Churchill Livingstone Limited, Edinburgh, London and New York.

4. Cruickshank, R., Duguid, J.P., Marimion, B.P., and Swain, R.H. (1980). Medical Microbiology. E.L.B.S., 12 ${ }^{\text {th }}$ Ed, Vol. 11, Reprinted Churchill Livingstone and Robert Stevenson, Edinburgh, EHI, 3AF.

5. Elkhayat, E.S., Alorainy, M.S., EL-Ashmawy, I.M., andFathi, S. (2016). Potential Antidepressant Constituents of Nigella sativa Seeds. Pharmacogn Mag.12(Suppl 1):S27-31.

6. Geller, R.J., Lopez, G.P., Cutler, S., Lin, D., Bachman, G.F., and Gorman, S.E. (2003). Atropine availability as an antidote for nerve agent casualties: Validated rapid reformulation of high-concentration atropine from bulk powder. Annals of Emergency Medicine. 41 (4): 453-6.

7. Ghoniem, M.A., EL-Khatib, E.N., andAttiaa, M.Z. (2001). Biochemical disorders in serum of Tilapia Nilotica exposed to diazinon for medium term. Vet. Med. J., Giza. 49(1):131-146.

8. Goldberg, D.M., and Spooner, R.J. (1983). Methods of enzymatic analysis (Bergmeyen, HV Ed.) $3^{\text {rd }}$ edn. Vol3, 258-265, verlogchemie, Deerfield beach, Fl.

9. HammadShafiq, Asif Ahmad, TariqMasud, andMuhammadKaleem. (2014). Cardio-protective and anti-cancer therapeutic potential of Nigella sativa. Iran J Basic Med Sci. 17(12): 967-979.

10. Hansch, C., Leo, A., andHoekman, D. (1995). Exploring QSAR: Volume 2: Hydrophobic, Electronic, and Steric Constants. Washington, DC: American Chemical Society. p. 106. ISBN 978-0-8412-2991-4.

11. Heremans, J.P., and Masson, P.L. (1973). Clin. Chem. 19:294-300.

12. Herigstad, B., Hamilton, M., andHeersink, J. (2001). How to optimize the drop plate method for enumerating bacteria. J. Microbiol. Meth. 44(2):121-129.

13. Hogg, N. (1998). Free radicals in disease. SeminReprodEndocrinol;16:241-248.

14. Levesque, (2007). SPSS Programming and Data Management: A Guide for SPSS and SAS Users, Fourth Edition (2007), SPSS Inc., Chicago Ill.

15. Mariod, A.A., Ibrahim, R.M., Ismail, M., and Ismail N. (2009). Antioxidant activity and phenolic content of phenolic rich fractions obtained from black cumin (Nigella sativa) seedcake. Food Chem116(1):306-312.

16. Maxwell, S.R. (1999). Antioxidant vitamin supplements: update of their potential benefits and possible risks. Drug Saf;21:253-266.

17. Meral, I., Yener, Z., Kahraman, T., andMert,N. (2001). Effect of Nigella sativa on Glucose Concentration, Lipid Peroxidation, Anti-Oxidant Defence System and Liver Damage in Experimentally Induced Diabetic Rabbits. J. Vet. Med. A48: 593-599.

18. Nishikimi, M., Roa, N.A., and Yogi, K. (1972). Biochem. Bioph. Res. Coomon. 46: 849-854.

19. Paglia, D.E., and Valentine, W.N. (1967). J. Lab. Clin. Med. 70: 158-169. 
20. Periasamy, V.S., Athinarayanan, J., andAlshatwi, A.A. (2016). Anticancer activity of an ultrasonic nanoemulsion formulation of Nigella sativa L. essential oil on human breast cancer cells. UltrasonSonochem31:449-55.

21. Sahak, M.K., Kabir, N., Abbas, G., Draman, S., Hashim, N.H., andHasanAdli, D.S.(2016). The Role of Nigella sativa and Its Active Constituents in Learning and Memory. Evid Based Complement Alternat Med.2016:60756079 .

22. Salem, M.L. (2005).Immunomodulatory and therapeutic properties of the Nigella sativa L. seed. IntImmunopharmacol5(13-14):1749-1770.

23. Schulz, J.B., Lindenau, J., Seyfried, J., Dichgans, J. (2000). Glutathione, oxidative stress and neurodegeneration. Eur J Biochem;267:4904-4911.

24. Umar, S., Zargan, J., Umar, K., Ahmad, S., Katiyar, C.K., and Khan, H.A. (2012). Modulation of the oxidative stress and inflammatory cytokine response by thymoquinone in the collagen induced arthritis in Wistar rats. ChemBiol Interact 197(1):40-46.

25. Young, D.S. (1995). Effects of drugs on clinical lab. Tests, 4th ed, AACC Press.

26. Young, D.S. (2001). Effects of disease on clinical lab. Tests, 4th ed, AACC Press.

27. Zelver, N., Hamilton, M., Pitts, B., Goeres, D., Walker, D., Sturman, P., andHeersink, J. (1999). Measuring antimicrobial effects on biofilm bacteria: in R. J. Doyle, et al. (eds), biofilm: methods in enzymology. Academic Press, San Diego, CA, pp. 608-628. 